\title{
Effect of arsenic and manganese on root growth and cell division in root tip cells of green gram (Vigna radiata $\mathrm{L}$.
}

\author{
S. Mumthas ${ }^{1 *}$, Al. A. Chidambaram ${ }^{2}$, P. Sundaramoorthy ${ }^{2}$ and \\ K. Sankar Ganesh ${ }^{3}$ \\ ${ }^{1}$ Peet Memorial Training College, Mavelikkara, Alappuzha, Kerala, India; ${ }^{2}$ Department of \\ Botany, Annamalai University, Annamalainagar, Tamilnadu, India; ${ }^{3}$ Post Graduate and \\ Research Department of Botany, Arignar Anna Arts College, \\ Villupuram, Tamilnadu, India
}

\begin{abstract}
Heavy metals are the metals having a density at least five times more than that of water. They are normally regarded as ones having an atomic number of 22-92. The effects of different concentration $(5,10,25,50$ and $100 \mathrm{mg} / \mathrm{l})$ of arsenic and manganese on germination, root growth and cell division in root tips of green gram (Vigna radiata.L) were studied. The inhibition of germination and root growth was noticed at higher concentrations of Arsenic and manganese. Arsenic had more toxic effect than manganese on the root tip cells of greengram during mitosis. Chromosome stickness implied the high toxicity of arsenic and manganese. The results also indicated that the germination percentage and root length was gradually decreased with the increasing concentration of both heavy metals.
\end{abstract}

Keywords: Arsenic, manganese, cell division, growth.

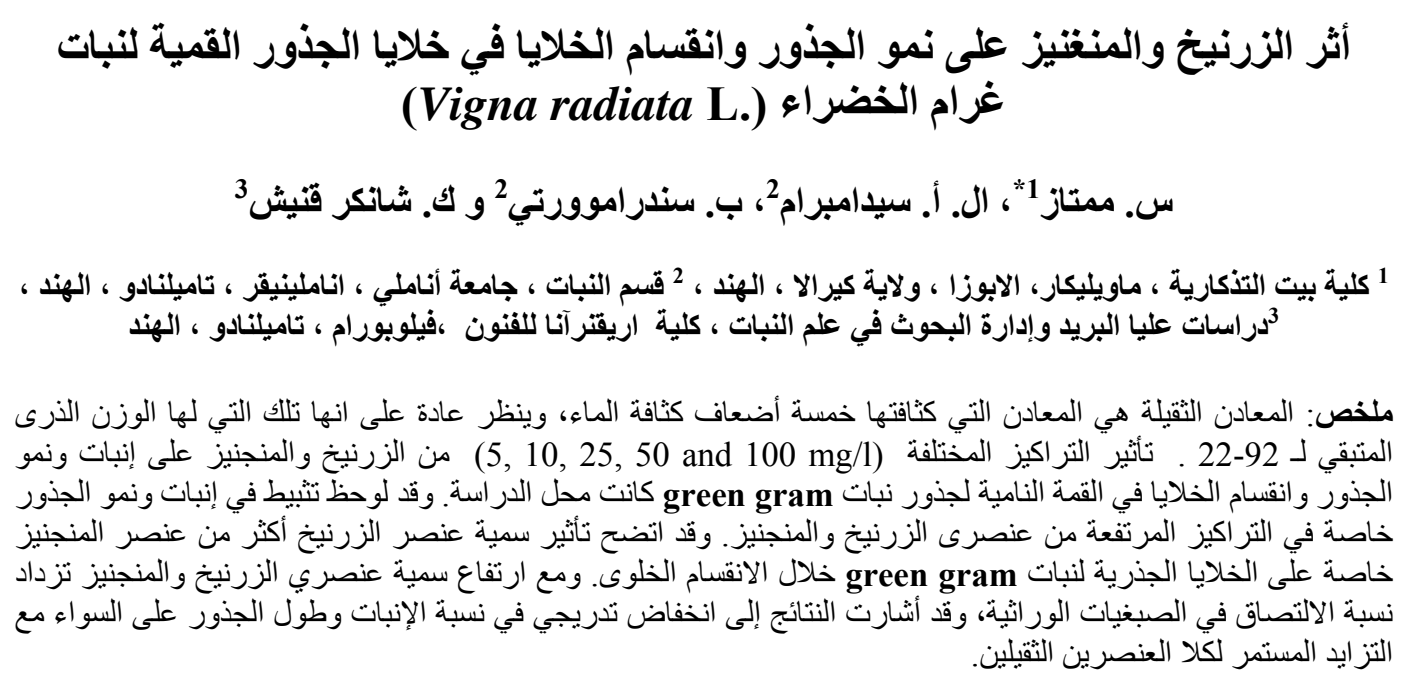

\footnotetext{
*Corresponding Author, Email: meenumumthas@gmail.com
} 


\section{Introduction}

Environment is the combination of external physical conditions that affect and influence the growth, development, and survival of an organism, including air, water and soil. In other words it is the combination of all the conditions external to the genome that potentially affect its expression and its structure. Among these, the problem of water pollution is getting greater dimension day by day in India. Water pollution is defined as the addition of any thing to water which alters the natural quality. Water is mostly polluted by the industrial wastewaters released from various industries. Heavy metals are the main constituents of many industrial effluents. The industrial, agricultural and municipal wastes are the key sources of these toxic heavy metals in the wastewater (Kirupalakshmi, 2004).

Heavy metals are the metals having a density at least five times more than that of water. They are normally regarded as ones having an atomic number of 22-92. Their common feature in relation to biological life is that in excess quantities they are poisonous and cause death of most living organisms. They can neither be created nor destroyed or any one heavy metal can be transformed into another. It therefore, means that once a metal is mobilized in the environment, its total amount remains the same (Sankar Ganesh, 2008).

Metal contamination issues are becoming increasingly common in elsewhere. Metals are natural part of terrestrial systems occurring in soil, rocks, air and water. A few metals including $\mathrm{Cu}$, $\mathrm{Mn}$ and $\mathrm{Zn}$ are however essential to plant metabolism in trace amounts. It is only when metals are present in bio available forms at excessive levels that they have the potentiality to become toxic to plants. Metal toxicity issues in plants and soils are a significant problem through out the world. Most metal toxicity occurs as a result of anthropogenic disturbance, such as mining, where unnaturally high amounts of metals are released during various processes.

When this polluted water is used for irrigation, they greatly affect the growth and productivity of the crops. Chromium, cadmium and copper are the most toxic metals to plants which result in reduced roots, phytomass and photosynthetic pigments, stunted growth and plant death eventually by interfering in many biochemical processes of the plant (Sankar Ganesh et al., 2006, 2008; Amico et al., 2008; Singh et al., 2008).

In recent years, land application of sewage sludge has become a common practice in many countries. The sludge depending on its origin may be rich in organic matter and nutritional elements as well as useless and toxic heavy metals (Singh and Keefer, 1989). Hence disposal of sewage waste into the land is highly recommended as it increases the fertility of the soil. However its indiscriminate application to the soil may increase the accumulation of heavy metals over the soil (Williams et al., 1980). One of the major concerns is the accumulation of heavy metals in edible parts of the crops creating hazards to animal and human health. Hence it would be worthwhile to undertake a study on the effects of heavy metals on the agricultural crops. The present investigation has been carried out to find out the effect of different concentrations of arsenic and manganese on seed germination, root growth and cytological studies of greengram (Vigna radiata (L.) Wilczek) var. Vamban 1.

\section{Materials and Methods}

The experimental plant greengram belongs to the family Fabaceae. It is one of the important pulse crops in India. The seeds of greengram were obtained from the National Pulses Research Centre, Vamban, Pudukkottai district of Tamil Nadu. Seeds of uniform in size, colour and weight were choosen for experimental purpose. 


\section{Laboratory experiments}

Sodium arsenate $\left(\mathrm{Na}_{2} \mathrm{HASO}_{4}-7 \mathrm{H}_{2} \mathrm{O}\right)$ salt is used as arsenic source for the present study. A known weight $(2.66 \mathrm{~g})$ of sodium arsenate was dissolved in $1000 \mathrm{ml}$ of distilled water to obtain the standard solution. From the standard solution, the different concentrations $(5,10,25,50$ and $100 \mathrm{mg} / \mathrm{l})$ of arsenic were prepared and used for the germination studies. Manganous sulphate $\left(\mathrm{MnSO}_{4} \mathrm{H}_{2} \mathrm{O}\right)$ salt is used as manganese source for the present study. The different concentrations $(5,10$, 25,50 and $100 \mathrm{mg} / \mathrm{l}$ ) of manganese were prepared by dissolving a known weight $(2.92 \mathrm{~g})$ of manganese sulphate and used for the germination studies.

\section{Germination studies}

The seeds were surface sterilized with $0.1 \%$ mercuric chloride solution for 2 minutes and washed thoroughly with tap water and then by distilled water for 30 minutes. The seeds were placed equispacially in sterilized petriplates lined with filter paper. Each petriplate was moistened uniformly by various concentrations of different heavy metal solutions. The seeds were irrigated with distilled water was treated as control. All the petriplates were kept under diffused sunlight at room temperature $\left(28 \pm 2{ }^{\circ} \mathrm{C}\right)$. The number of seeds germinated in each treatment was counted and calculated on $7^{\text {th }}$ day after sowing. The emergence of radicle was taken as a criterion for germination. Ten seedlings from each replicate were selected for recording the root length.

\section{Cytological studies}

Root tips were utilised for cytological investigation. Root tips from the greengram plants were collected, washed with distilled water and fixed in 1:3 acetic alcohol for 24 hours and then stored in $70 \%$ alcohol for subsequent use. The root tips were fixed between 8.45 am to $9.25 \mathrm{am}$. The greengram seeds were treated with various concentration of different heavy metal (arsenic and manganese) solutions $(5,10$, 25,50 and $100 \mathrm{mg} / \mathrm{l}$ ) in petriplates. After, two days the root tips were taken and used for cytological studies. The root tips collected from greengram which were not given the heavy metal treatment were taken as control.

The root tips after treatment were washed in distilled water and fixed in 1:3 acetic alcohol. They were kept for over night in the fixative and were stored in $70 \%$ alcohol for subsequent use. Then root tip squashes were made by using iron alum, haematoxylin squash technique of Marimuthu and Subramaniam (1960). This haematoxylin squash technique was found to be suitable for the cytological investigation.

\section{Results}

In the present study, seed germination percentage and root growth decreased gradually with increase in heavy metals (Arsenic and manganese) concentrations. The control plants exhibit maximum percentage of germination and root growth when compared with all other concentrations (Table 1). The detailed study of chromosome morphology, size, type and number were observed in 5, 10, $25, \quad 50$ and $100 \mathrm{mg} / \mathrm{l}$ of arsenic concentration. In these cases, it showed the chromosomal aberrations, which revealed the diploid complement of chromosome $2 \mathrm{n}$ $=2$. The total chromosome length $(75.2$ $\mu \mathrm{m})$, absolute chromosome length (37.6 $\mu \mathrm{m})$ and average chromosome length (3.41 $\mu \mathrm{m})$ was observed at $5 \mathrm{mg} / \mathrm{l}$. The total chromosome length $(68.1 \mu \mathrm{m})$, absolute chromosome length $(34.05 \mu \mathrm{m})$ and average chromosome length $(3.11 \mu \mathrm{m})$ was recorded in $10 \mathrm{mg} / \mathrm{l}$. The total chromosome length concentrations $(65.4 \mu \mathrm{m}$, absolute chromosome length $(32.7 \mu \mathrm{m})$ and average chromosome length $(2.97 \mu \mathrm{m})$ was recorded in $25 \mathrm{mg} / \mathrm{l}$. The total chromosome length $(57.8 \mu \mathrm{m})$, absolute chromosome length $(28.9 \mu \mathrm{m})$ and average chromosome 
length $(2.63 \mu \mathrm{m})$ were recorded in $50 \mathrm{mg} / \mathrm{l}$. The total chromosome length $(51.7 \mu \mathrm{m})$, absolute chromosome length $(25.85 \mu \mathrm{m})$ and average chromosome length $(2.35 \mu \mathrm{m})$ was observed in $100 \mathrm{mg} / 1$ concentrations (Table 2, 3 and Figure 1).

Table 1. Effect of arsenic and manganese on germination and root length of greengram (Vigna radiata (L.) Wilczek).

\begin{tabular}{lllll}
\hline $\begin{array}{l}\text { Heavy Metal } \\
\text { Concentrations } \\
\text { (Mg/l) }\end{array}$ & Arsenic & \multicolumn{3}{c}{ Manganese } \\
\cline { 2 - 5 } & Germination \% & $\begin{array}{l}\text { Root Length } \\
\text { (cm/seedling) }\end{array}$ & Germination \% & $\begin{array}{l}\text { Root Length } \\
\text { (cm/seedling) }\end{array}$ \\
\hline Control & $95.0 \pm 4.75$ & $8.70 \pm 0.435$ & $95.0 \pm 4.75$ & $8.70 \pm 0.435$ \\
5 & $81.2 \pm 4.06$ & $5.34 \pm 0.267$ & $90.0 \pm 4.50$ & $6.00 \pm 0.3$ \\
10 & $73.0 \pm 3.65$ & $4.20 \pm 0.210$ & $84.0 \pm 4.20$ & $5.50 \pm 0.275$ \\
25 & $62.0 \pm 3.10$ & $3.04 \pm 0.152$ & $73.0 \pm 3.65$ & $4.74 \pm 0.237$ \\
50 & $45.0 \pm 2.25$ & $2.20 \pm 0.110$ & $60.0 \pm 3.00$ & $4.50 \pm 0.225$ \\
100 & $35.0 \pm 1.75$ & $1.84 \pm 0.092$ & $50.0 \pm 2.50$ & $2.94 \pm 0.147$ \\
\hline
\end{tabular}

\pm Standard deviation; No germination was recorded beyond $100 \mathrm{mg} / \mathrm{l}$

The effect of different concentrations of manganese in greengram seedlings showed the following numerical aberrations in the diploid complement. In this case, the metaphase chromosome revealed the diploid complement with $2 \mathrm{n}=$ 22 chromosomes. The total chromosome length in $(78.7 \mu \mathrm{m})$, absolute chromosome length $(39.35 \mu \mathrm{m})$ and average chromosome length $(3.6 \mu \mathrm{m})$ were observed in $5 \mathrm{mg} / \mathrm{l}$. The total chromosome length $(73.1 \mu \mathrm{m})$, absolute chromosome length $(36.55 \mu \mathrm{m})$ and average chromosome length $(3.3 \mu \mathrm{m})$ were recorded in $10 \mathrm{mg} / \mathrm{l}$. The total chromosome length (71.4 $\mu \mathrm{m})$, absolute chromosome length $(35.7 \mu \mathrm{m})$ and average chromosome length $(3.2 \mu \mathrm{m})$ was recorded in $25 \mathrm{mg} / \mathrm{l}$. The total chromosome length $(67.5 \mu \mathrm{m})$, absolute chromosome length $(33.75 \mu \mathrm{m})$ and average chromosome length $3 \mu \mathrm{m}$ were recorded in $25 \mathrm{mg} / 1$ concentration. The total chromosome length $(65.5 \mu \mathrm{m})$, absolute chromosome length $(28.25 \mu \mathrm{m})$ and average chromosome length $(2.57 \mu \mathrm{m})$ were recorded in $100 \mathrm{mg} / \mathrm{l}$ concentrations (Table 4, 5 and Figure 2).

The different concentrations (5-100 $\mathrm{mg} / \mathrm{l}$ ) of heavy metals on greengram seedlings showed different spectrum of abnormal cells and frequency of abnormalities. The abnormal cells in the control plant showed less in number (5) and frequency (1.92). This was followed by the successive concentrations $(5,10,25$, 50 and $100 \mathrm{mg} / \mathrm{l}$ ) of various heavy metals. It was revealed that number of abnormal cells increased with increased in concentration of heavy metals. Among the heavy metals, arsenic showed more toxic effect than manganese. So, the higher (100 $\mathrm{mg} / \mathrm{l}$ ) concentrations of arsenic provided more number of abnormal cells (25) and frequency (11.74) than that of the other concentrations and the control. 
Emir. J. Food Agric. 2010. 22 (4): 285-297

http://ffa.uaeu.ac.ae/ejfa.shtml

Table 2. Effect of different concentrations of arsenic on cytological behaviour of greengram (Vigna radiata (L.) Wilczek).

\begin{tabular}{|c|c|c|c|c|c|c|c|c|c|}
\hline \multirow{2}{*}{$\begin{array}{l}\text { Conc. of } \\
\text { Arsenic } \\
(\mathrm{mg} / \mathrm{l}) \\
\end{array}$} & \multicolumn{2}{|c|}{ Chromosome } & \multicolumn{4}{|c|}{ Chromosome length in $\mu \mathrm{m}$} & \multirow[b]{2}{*}{ L/S ratio } & \multirow{2}{*}{$\begin{array}{l}\text { Position of } \\
\text { centromere }\end{array}$} & \multirow[b]{2}{*}{ Chromosome length } \\
\hline & Type & No. & $\begin{array}{l}\text { Long arm } \\
\text { (L) }\end{array}$ & $\begin{array}{l}\text { Short arm } \\
\text { (S) }\end{array}$ & $\begin{array}{l}\text { Satellite } \\
\text { (S) }\end{array}$ & $\begin{array}{l}\text { Total length } \\
(\mathbf{L}+\mathbf{S}+\mathbf{S})\end{array}$ & & & \\
\hline \multirow{5}{*}{ Control } & $\mathrm{J}$ & 3 & 3.9 & 2.7 & - & 20.4 & 1.4 & Submedian & \multirow{5}{*}{$\begin{array}{l}\text { Total chromosome length }=80.5 \mu \mathrm{m} \\
\text { Absolute chromosome length }=40.25 \mu \mathrm{m} \\
\text { Average chromosome length }=3.66 \mu \mathrm{m}\end{array}$} \\
\hline & $\mathrm{V}$ & 7 & 1.9 & 1.9 & - & 28 & 1 & Median & \\
\hline & $\mathrm{S}$ & 2 & 4.4 & 3.3 & 0.2 & 16.2 & 1.3 & Submedian & \\
\hline & I & 6 & 1.8 & 0.2 & - & 13.2 & 9 & Subterminal & \\
\hline & I & 4 & 1.5 & 0.2 & - & 9.5 & 7.5 & subterminal & \\
\hline \multirow{5}{*}{5} & $\mathrm{~J}$ & 3 & 3.9 & 3 & - & 14.6 & 1.36 & Submedian & \multirow{5}{*}{$\begin{array}{l}\text { Total chromosome length }=68.9 \mu \mathrm{m} \\
\text { Absolute chromosome length }=34.45 \mu \mathrm{m} \\
\text { Average chromosome length }=3.13 \mu \mathrm{m}\end{array}$} \\
\hline & $\mathrm{S}$ & 2 & 3.8 & 2.3 & 0.2 & 13.4 & 1.52 & Submedian & \\
\hline & $\mathrm{V}$ & 8 & 1.5 & 1.5 & - & 25.6 & 1 & Median & \\
\hline & I & 6 & 1.2 & 0.2 & - & 10.2 & 6.5 & Subterminal & \\
\hline & I & 3 & 1.3 & 0.2 & - & 5.1 & 6.5 & Subterminal & \\
\hline \multirow{5}{*}{10} & $\mathrm{~S}$ & 2 & 3.5 & 2.1 & 0.2 & 12 & 1.68 & Submedian & \multirow{5}{*}{$\begin{array}{l}\text { Total chromosome length }=68.1 \mu \mathrm{m} \\
\text { Absolute chromosome length }=34.05 \mu \mathrm{m} \\
\text { Average chromosome length }=3.11 \mu \mathrm{m}\end{array}$} \\
\hline & $\mathrm{J}$ & 3 & 3.7 & 2.4 & - & 18.9 & 1.44 & Submedian & \\
\hline & $\mathrm{V}$ & 7 & 1.3 & 1.3 & - & 19.6 & 1 & Median & \\
\hline & I & 6 & 1.4 & 0.2 & - & 10.8 & 7 & Subterminal & \\
\hline & I & 4 & 1.3 & 0.2 & - & 6.8 & 6.5 & Subterminal & \\
\hline \multirow{5}{*}{25} & $\mathrm{~S}$ & 2 & 3.1 & 2.0 & 0.2 & 11 & 1.55 & Submedian & \multirow{5}{*}{$\begin{array}{l}\text { Total chromosome length }=65.4 \mu \mathrm{m} \\
\text { Absolute chromosome length }=32.7 \mu \mathrm{m} \\
\text { Average chromosome length }=2.97 \mu \mathrm{m}\end{array}$} \\
\hline & $\mathrm{J}$ & 3 & 3.2 & 2.3 & - & 17.1 & 1.39 & Submedian & \\
\hline & $\mathrm{V}$ & 8 & 1.3 & 1.3 & - & 22.4 & 1 & Median & \\
\hline & I & 5 & 1.3 & 0.2 & - & 8.5 & 6.5 & Subterminal & \\
\hline & I & 4 & 1.2 & 0.2 & - & 6.4 & 6 & Subterminal & \\
\hline \multirow{5}{*}{50} & $\mathrm{~S}$ & 2 & 2.9 & 1.9 & 0.2 & 10.4 & 1.53 & Submedian & \multirow{5}{*}{$\begin{array}{l}\text { Total chromosome length }=57.8 \mu \mathrm{m} \\
\text { Absolute chromosome length }=28.9 \mu \mathrm{m} \\
\text { Average chromosome length }=2.63 \mu \mathrm{m}\end{array}$} \\
\hline & $\mathrm{J}$ & 2 & 3.0 & 2.1 & - & 10.6 & 1.43 & Submedian & \\
\hline & $\mathrm{V}$ & 8 & 1.2 & 1.2 & - & 20.8 & 1 & Median & \\
\hline & I & 6 & 1.2 & 0.2 & - & 9.6 & 6 & Subterminal & \\
\hline & I & 4 & 1.2 & 0.2 & - & 6.4 & 6 & Subterminal & \\
\hline \multirow{5}{*}{100} & $\mathrm{~S}$ & 2 & 2.2 & 1.7 & 0.2 & 8.6 & 1.3 & Submedian & \multirow{5}{*}{$\begin{array}{l}\text { Total chromosome length }=51.7 \mu \mathrm{m} \text { Absolute } \\
\text { chromosome length }=25.85 \mu \mathrm{m} \\
\text { Average chromosome length }=2.35 \mu \mathrm{m}\end{array}$} \\
\hline & $\mathrm{J}$ & 3 & 2.8 & 1.9 & - & 14.7 & 1.47 & Submedian & \\
\hline & $\mathrm{V}$ & 8 & 0.9 & 0.9 & - & 16 & 1 & Median & \\
\hline & I & 5 & 0.8 & 0.2 & - & 6 & 4 & Subterminal & \\
\hline & I & 4 & 1.2 & 0.2 & - & 6.4 & 6 & Subterminal & \\
\hline
\end{tabular}


Table 3. Effect of different concentrations of arsenic (mg/l) in greengram (Vigna radiata (L.) Wilczek) on total number of abnormal cells, frequency of abnormalities and percentage of mitotic abnormalities.

\begin{tabular}{|c|c|c|c|c|c|c|c|c|c|c|c|}
\hline \multirow{2}{*}{$\begin{array}{l}\text { Concentration } \\
\text { of Arsenic } \\
(\mathrm{mg} / \mathrm{l})\end{array}$} & \multirow{2}{*}{$\begin{array}{l}\text { Total No. } \\
\text { of cells } \\
\text { analysed }\end{array}$} & \multirow{2}{*}{$\begin{array}{l}\text { Total no. of } \\
\text { abnormal } \\
\text { cells }\end{array}$} & \multicolumn{4}{|c|}{ Number of abnormal cells } & \multirow{2}{*}{$\begin{array}{l}\text { Frequency } \\
\text { of total } \\
\text { abnormalities }\end{array}$} & \multicolumn{4}{|c|}{$\%$ of mitotic abnormalities } \\
\hline & & & Bridge & Laggard & Stickiness & $\begin{array}{l}\text { Binucleate } \\
\text { cells }\end{array}$ & & Bridge & Laggard & Stickiness & $\begin{array}{l}\text { Binucleate } \\
\text { cells }\end{array}$ \\
\hline Control & 260 & 5 & 2 & 1 & 1 & 1 & 1.92 & 0.77 & 0.38 & 0.38 & 0.38 \\
\hline 5 & 256 & 12 & 5 & 3 & 2 & 2 & 4.69 & 2 & 1.17 & 0.78 & 0.78 \\
\hline 10 & 243 & 17 & 7 & 5 & 3 & 2 & 7 & 7 & 2.05 & 1.23 & 0.82 \\
\hline 25 & 231 & 19 & 9 & 5 & 3 & 3 & 8.2 & 8.2 & 2.16 & 1.3 & 1.29 \\
\hline 50 & 224 & 23 & 7 & 9 & 4 & 3 & 10.27 & 10.27 & 4.02 & 1.8 & 1.3 \\
\hline 100 & 213 & 25 & 9 & 7 & 5 & 4 & 11.74 & 11.7 & 3.29 & 2.35 & 1.9 \\
\hline
\end{tabular}


Emir. J. Food Agric. 2010. 22 (4): 285-297

http://ffa.uaeu.ac.ae/ejfa.shtml
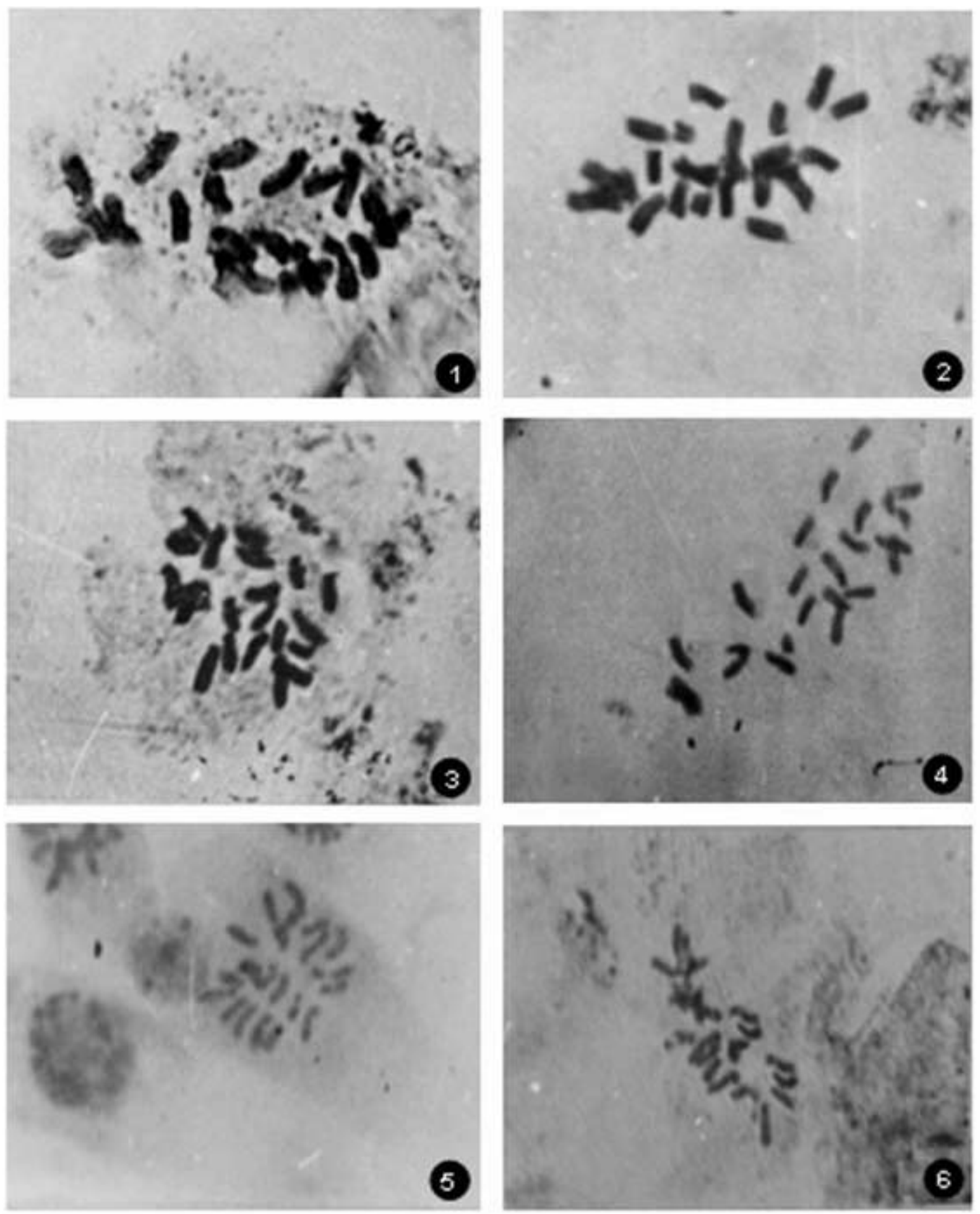

Figure 1. Effect of Arsenic on mitosis of green gram Mitosis (Arsenic). Microphotographs $\times$ 1250. Figure 1. 1. Control; $2 \mathrm{n}=22$; Figure 1.2. $5 \mathrm{mg} / \mathrm{l} ; 2 \mathrm{n}=22$; Figure $1.3 .10 \mathrm{mg} / \mathrm{l} ; 2 \mathrm{n}=22$;

Figure 1.4. $25 \mathrm{mg} / \mathrm{l} ; 2 \mathrm{n}=22$; Figure 1.5. $50 \mathrm{mg} / \mathrm{l} ; \quad 2 \mathrm{n}=22$; Figure 1.6. $100 \mathrm{mg} / \mathrm{l} ; 2 \mathrm{n}=22$ 
Table 4. Effect of different concentrations of manganese on cytological behaviour of greengram (Vigna radiata (L.) Wilczek) .

\begin{tabular}{|c|c|c|c|c|c|c|c|c|c|}
\hline \multirow{2}{*}{$\begin{array}{l}\text { Concentration } \\
\text { of Manganese } \\
(\mathrm{mg} / \mathrm{l})\end{array}$} & \multicolumn{2}{|c|}{ Chromosome } & \multicolumn{4}{|c|}{ Chromosome length in $\mu \mathrm{m}$} & \multirow[b]{2}{*}{$\mathrm{L} / \mathrm{S}$ ratio } & \multirow[b]{2}{*}{$\begin{array}{l}\text { Position of } \\
\text { centromere }\end{array}$} & \multirow[b]{2}{*}{ Chromosome length } \\
\hline & Type & No. & $\begin{array}{l}\text { Long arm } \\
\text { (L) }\end{array}$ & $\begin{array}{l}\text { Short arm } \\
\text { (S) }\end{array}$ & $\begin{array}{l}\text { Satellite } \\
\text { (S) }\end{array}$ & $\begin{array}{l}\text { Total length } \\
(\mathbf{L}+\mathbf{S}+\mathbf{S})\end{array}$ & & & \\
\hline \multirow{5}{*}{ Control } & $\mathrm{J}$ & 3 & 3.9 & 2.7 & - & 20.4 & 1.4 & Submedian & Total chromosome length $=80.5$ \\
\hline & $\mathrm{V}$ & 7 & 1.9 & 1.9 & - & 28 & 1 & Median & $\mu \mathrm{m}$ \\
\hline & $\mathrm{S}$ & 2 & 4.4 & 3.3 & 0.2 & 16.2 & 1.3 & Submedian & Absolute chromosome length $=40.25$ \\
\hline & $\mathrm{I}$ & 6 & 1.8 & 0.2 & - & 13.2 & 9 & Subterminal & $\mu \mathrm{m}$ \\
\hline & I & 4 & 1.5 & 0.2 & - & 9.5 & 7.5 & subterminal & $\begin{array}{l}\text { Average chromosome length }=3.66 \\
\mu \mathrm{m}\end{array}$ \\
\hline \multirow{5}{*}{5} & $\mathrm{~S}$ & 2 & 4 & 3 & 0.2 & 14.8 & 1.33 & Submedian & Total chromosome length $=78.7 \mu \mathrm{m}$ \\
\hline & $\mathrm{J}$ & 3 & 3.7 & 2.5 & - & 19.2 & 1.48 & Submedian & Absolute chromosome length $=39.35$ \\
\hline & $\mathrm{V}$ & 7 & 1.7 & 0.2 & - & 25.2 & 1 & Median & $\mu \mathrm{m}$ \\
\hline & I & 5 & 1.6 & 0.2 & - & 10 & 8 & Subterminal & Average chromosome length $=3.6$ \\
\hline & I & 5 & 1.5 & 0.2 & - & 9.5 & 7.5 & Subterminal & $\mu \mathrm{m}$ \\
\hline \multirow{5}{*}{10} & $\mathrm{~S}$ & 2 & 3.7 & 2.8 & 0.2 & 13.8 & 1.32 & Submedian & Total chromosome length $=73.1 \mu \mathrm{m}$ \\
\hline & $\mathrm{J}$ & 3 & 3.6 & 2.2 & - & 18 & 1.6 & Submedian & Absolute chromosome length $=36.55$ \\
\hline & $\mathrm{V}$ & 6 & 1.7 & 1.7 & - & 21.6 & 1 & Median & $\mu \mathrm{m}$ \\
\hline & $\mathrm{I}$ & 5 & 1.5 & 0.2 & - & 9.5 & 7.5 & Subterminal & Average chromosome length $=3.3$ \\
\hline & I & 6 & 1.3 & 0.2 & - & 10.2 & 6.5 & Subterminal & $\mu \mathrm{m}$ \\
\hline \multirow{5}{*}{25} & $\mathrm{~S}$ & 2 & 3.3 & 2.5 & 0.2 & 12.4 & 1.17 & Submedian & Total chromosome length $=71.4 \mu \mathrm{m}$ \\
\hline & $\mathrm{J}$ & 3 & 3.5 & 2.7 & - & 19.2 & 1.37 & Submedian & Absolute chromosome length $=35.7$ \\
\hline & $\mathrm{V}$ & 6 & 1.6 & 1.6 & - & 20.4 & 1 & Median & $\mu \mathrm{m}$ \\
\hline & I & 6 & 1.5 & 0.2 & - & 11.4 & 2.14 & Subterminal & Average chromosome length $=3.2$ \\
\hline & I & 5 & 1.2 & 0.2 & - & 8 & 3 & Subterminal & $\mu \mathrm{m}$ \\
\hline \multirow{5}{*}{50} & $\mathrm{~S}$ & 2 & 3.0 & 2.1 & 0.2 & 10.6 & 1.43 & Submedian & \multirow{5}{*}{$\begin{array}{l}\text { Total chromosome length }=67.5 \mu \mathrm{m} \\
\text { Absolute chromosome length }=33.75 \\
\mu \mathrm{m} \\
\text { Average chromosome length }=3 \mu \mathrm{m}\end{array}$} \\
\hline & $\mathrm{J}$ & 3 & 3.2 & 1.9 & - & 15.9 & 1.68 & Submedian & \\
\hline & $\mathrm{V}$ & 8 & 1.5 & 1.5 & - & 25.6 & 1 & Median & \\
\hline & I & 5 & 1.4 & 0.2 & - & 9 & 7 & Subterminal & \\
\hline & $\mathrm{I}$ & 4 & 1.3 & 0.1 & - & 6.4 & 13 & Subterminal & \\
\hline \multirow{5}{*}{100} & $\mathrm{~S}$ & 2 & 2.5 & 1.9 & 0.2 & 9.6 & 1.32 & Submedian & Total chromosome length $=56.5 \mu \mathrm{m}$ \\
\hline & $\mathrm{J}$ & 3 & 3 & 2.1 & - & 15.9 & 1.43 & Submedian & Absolute chromosome length $=28.25$ \\
\hline & $\mathrm{V}$ & 7 & 1.1 & 1.1 & - & 16.8 & 1 & Median & $\mu \mathrm{m}$ \\
\hline & $\mathrm{I}$ & 6 & 0.9 & 0.2 & - & 7.8 & 4.5 & Subterminal & Average chromosome length $=2.57$ \\
\hline & $\mathrm{I}$ & 4 & 1.3 & 0.1 & - & 6.4 & 13 & Subterminal & $\mu \mathrm{m}$ \\
\hline
\end{tabular}


Emir. J. Food Agric. 2010. 22 (4): 285-297

http://ffa.uaeu.ac.ae/ejfa.shtml

Table 5. Effect of different concentrations of manganese ( $\mathrm{mg} / \mathrm{l})$ in greengram (Vigna radiata (L.) Wilczek) on total number of abnormal cells, frequency of abnormalities and percentage of mitotic abnormalities.

\begin{tabular}{|c|c|c|c|c|c|c|c|c|c|c|c|}
\hline \multirow{2}{*}{$\begin{array}{l}\text { Concentrations } \\
\text { of Manganese } \\
(\mathrm{mg} / \mathrm{l})\end{array}$} & \multirow{2}{*}{$\begin{array}{l}\text { Total No. } \\
\text { of cells } \\
\text { analysed }\end{array}$} & \multirow{2}{*}{$\begin{array}{l}\text { Total no. of } \\
\text { abnormal } \\
\text { cells }\end{array}$} & \multicolumn{4}{|c|}{ Number of abnormal cells } & \multirow{2}{*}{$\begin{array}{l}\text { Frequency of } \\
\text { total } \\
\text { abnormalities }\end{array}$} & \multicolumn{4}{|c|}{$\%$ of mitotic abnormalities } \\
\hline & & & Bridge & Laggard & Stickiness & $\begin{array}{l}\text { Binucleate } \\
\text { cells }\end{array}$ & & Bridge & Laggard & Stickiness & $\begin{array}{l}\text { Binucleate } \\
\text { cells }\end{array}$ \\
\hline Control & 260 & 5 & 2 & 1 & 1 & 1 & 1.9 & 0.77 & 0.38 & 0.38 & 0.38 \\
\hline 5 & 253 & 10 & 5 & 3 & 2 & 1 & 4 & 2 & 1.19 & 0.79 & 4 \\
\hline 10 & 246 & 14 & 7 & 3 & 2 & 2 & 5.69 & 2.85 & 1.22 & 0.81 & 0.81 \\
\hline 25 & 232 & 17 & 8 & 4 & 3 & 2 & 7.33 & 3.4 & 1.72 & 1.3 & 0.86 \\
\hline 50 & 229 & 19 & 9 & 5 & 3 & 2 & 8.3 & 4.4 & 2.18 & 1.31 & 0.87 \\
\hline 100 & 218 & 21 & 11 & 5 & 3 & 2 & 9.6 & 5.04 & 2.29 & 1.37 & 0.92 \\
\hline
\end{tabular}



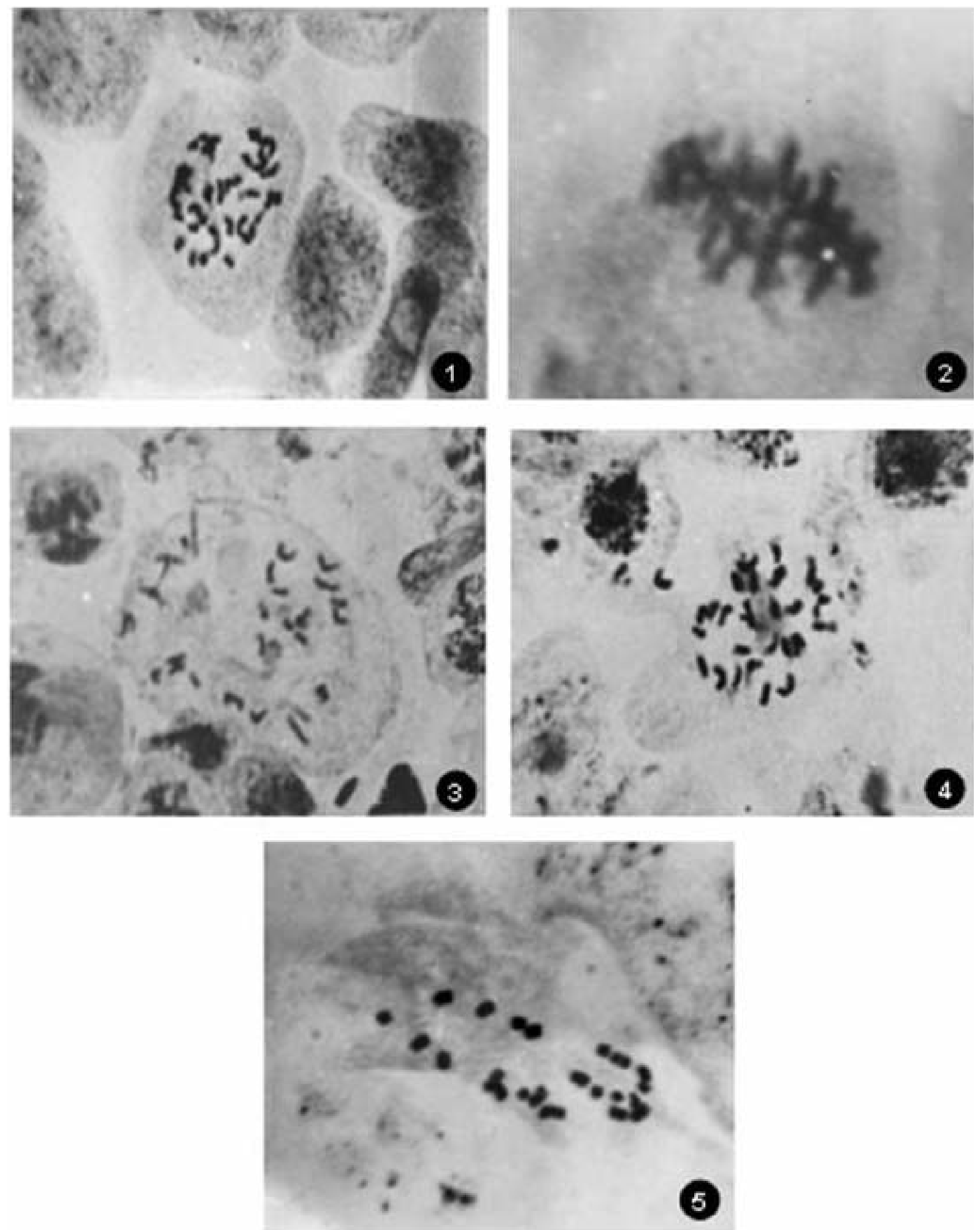

Figure 2. Effect of manganese on mitosis of green gram Mitosis (Manganese). Microphotographs $\times$ 1250. Figure 2.1. $5 \mathrm{mg} / \mathrm{l} ; 2 \mathrm{n}=22$, Figure 2.2. $10 \mathrm{mg} / \mathrm{l} ; 2 \mathrm{n}=22$, Figure $2.3 .25 \mathrm{mg} / \mathrm{l} ; 2 \mathrm{n}=22$, Figure 2.4. $50 \mathrm{mg} / \mathrm{l} ; 2 \mathrm{n}=22$, Figure $2.5 .100 \mathrm{mg} / \mathrm{l} ; 2 \mathrm{n}=22$. 


\section{Discussion}

Heavy metals are one of the most important groups of pollutants of aquatic environment, which originate from domestic sewage, industrial effluents and agricultural run off etc. Addition of heavy metals (As, $\mathrm{Al}, \mathrm{Cr}, \mathrm{Mn}, \mathrm{Mo}, \mathrm{Ni}, \mathrm{Cu}, \mathrm{Pb}$, etc.) into the environment causes toxic and carcinogenic effects on flora and fauna and create great ecological crisis at the global level. Heavy metal accumulation in soil and its importance on the morphological, biochemical and cytological aspects of plants have received more attention in recent times by many workers (Abbasi et al., 1992; Premkumar et al., 2001; Prakash et al., 2004).

The inhibition of germination and root growth was noticed at higher concentrations of Arsenic and manganese. Similar result was also reported in wheat (Panda and Patra, 1997), Cowpea (Lalitha et al., 1999), Pea (Chugh and Sawhney, 1996) and cotton (Shrivastava et al., 1997). The reduction in germination percentage of greengram at higher concentrations may be attributed to the interference of heavy metal ions during germination metabolism (Sankar Ganesh, 2008). Similarly, the reduction in root growth is possibly due to the accumulation of heavy metals in the plant tissues and its interaction with the minerals (Banu et al., 1997).

$$
\text { Rapid urbanization and }
$$
industrialization have enhanced levels of toxic heavy metals in the environment posing a potential health hazard for all living organisms. Heavy metals have some genotoxic potential. Growth inhibition at higher concentrations may be linked with lower mitotic activity in the root meristematic zone or to an inhibition of cell enlargement in the elongation zone as a consequence of decreased cellular turgor (Gabbrielli et al., 1990). From the cytological studies, the researcher can understand that the chromosomal aberrations are more in arsenic treatment and less in manganese treatment seedlings when compared to the control. All kinds of chromosomal variations are found to be increased with increase in heavy metal concentrations.

The formation of bridges could be attributed to chromosome stickiness and to chromosome breakage and reunion. The induction of lagging could be attributed to the failure of the normal organization and function of the spindle apparatus. Such type of abnormalities is due to the loss of microtubule of spindle fibers. The micronuclei observed at higher doses of both salts, may originate from a lagging chromosome or from a chromosome fragment. This was supported by previous reports of several authors (Patil and Bhat, 1992; Salam et al., 1993; Chidambaram et al., 2009). Nagpal and Grover (1994) classified induced chromosomal abnormalities into two groups viz., clastogenic effects such as fragments, ring chromosome bridges and micronuclei. The precocious movement of the chromosome might have been caused by the early terminalisation, stickiness of chromosome or because of the movement of the chromosome ahead of the rest during anaphase (Permjit and Grover, 1985; Chidambaram et al., 2009).

The cytological and genetic effects of some of the heavy metals like mercury, cadmium, chromium and lead in animal and plant cells have been studied by Sharma et al. (1988) and Zou et al. (2006). The most abundant of them were stickiness, breakage, lagging, bridges and disturbed phases at the level of $\mathrm{M}_{2}$ seed storage protein. The metal treatment caused changes in protein banding patterns especially at the high molecular weight regions (George, 1999). The chromosomal aberration like stickiness, laggards, chromosome bridges, irregular metaphase, fragmentation and binucleate cells were increased with the increasing concentrations of metals. Similar results were reported by Kumar and Tripathi (2003). 
The present study reveals that the presence of heavy metal, in the irrigated water causes many variations in germination, growth, biochemical and cytological behaviour of greengram. It can be concluded that the heavy metal containing wastewater are toxic to crops. So, these polluted water should be properly treated to remove the heavy metals and treated water with suitable dilution may be used for irrigation purpose.

\section{Acknowledgement}

The authors are thankful to Head of the Department of Botany, Annamalai University, Annamalainagar, Tamilnadu, India, for providing the laboratory facilities.

\section{References}

Abbasi, S., T. K. Kunjahamed, K. Mahadevan, P. C. Nipancy and R. Soni, 1992. Environmental management of chromium, copper and zinc with respect to impact on growth and germination of gram (Cicer arietinum). J. Inst. Public Hlth. Engras India 1:12-13.

Amico, E. D., L. Cavalca and V. Andreoni, 2008. Improvement of Brassica napus growth under cadmium stress by cadmium-resistant rhizobacteria. Soil Biol. Biochem. 40:74-84.

Banu, B., P. Thangavel and V. Subburam, 1997. Toxicity of neolan gray to Vigna radiata CO 3. Poll. Res. 16:2528.

Chidambaram. Al. A, P. Sundaramoorthy, A. Murugan, K. Sankar Ganesh and L. Baskaran. 2009. Chromium induced cytotoxicity in black gram(Vigna radiata. L), Iran. J. Health. Sci. Eng. 6(1):7-22.

Chugh, L. K. and S. K. Sawhney. 1996. Effect of cadmium on germination amylases and rate of pea respiration of germinating pea seeds. Environ. Poll., 19:107-124.

Gabbrielli, R., T. Pandolfini, O. Verganano and M. R. Palandrii. 1990. Comparison of two serpentine species with different nickel tolerance strategies. Plant Soil 122:27-277.

George, N. M. 1999. Evaluation on mutagenic effects of the three heavy metals on Vicia faba plants. Cytologia 75-81.

Kirupalakshmi, S., 2004. Biosorption of hexavalent chromium by marine Algae. Poll. Res. 4(23):737-739.

Kumar, G. and A. Tripathi, 2003. Comparative mitotoxicity and mutagenicity of two heavy metals in Allium cepa L. Cytol. Genet. 4:169173.

Lalitha, K., N. Balasubrahmanian and S. Kalavathy. 1999. Studies of impact of chromium on Vigna unguiculata (L.) Walp. var. (Long). J. Swamy Bot. 16:17-20.

Marimuthu, K. M. and M. K. Subramaniam. 1960. A haematoxylin squash method for the root tips of Dolichos lablab L. Curr. Sci. 29:482493.

Nagpal, A. and I. S. Grover. 1994. Genotoxic evaluation of systemic pesticides in Allium cepa L. mitotic effects. Nucleus 37:99-105.

Panda, S. K. and H. K. Patra. 1997. Physiology of chromium toxicity in plants. Rev. Plant Physiol. Biochem. 24(1):10-17.

Patil, B. C. and G. J. Bhat. 1992. A comparative study of $\mathrm{MH}$ and EMS in the induction of chromosomal 
aberration on lateral root meristem in Clittoria ternatia L. Cytologia 57:259-264.

Permjit, K. and I. S. Grover. 1985. Cytological effects of some organophosphorus pesticides II meiotic effect. Cytologia 50:199-211.

Prakash, S. M. M. S., V. R. Yogeeth, R. Parama and R. Gowda. 2004. Effect of irrigation water with chromium on germination and growth of radish (Raphanus sativus L.). Nature Environ. Poll. Chem. 26:881-883.

Premkumar, C., A. P. Nagaraju and S. B. Yogananda. 2001. Effect of phosphorus sources and zinc levels on growth and yield of cowpea (Vigna unguiculata). J. Ecobiol. 4:275-278.

Salam, H. Z., El-Abdin, Ebtissam, H. A. Hussein, El-Itriby, A. Hanaiya, Anwar, A. Wagida and S. A. Mansour. 1993. The mutagenicity of gramoxone (paraquat) on different eukaryotic system. Mutat. Res. 319:89-101.

Sankar Ganesh, K., Al. A. Chidambaram, P. Sundaramoorthy, L. Baskaran and M. Selvaraj. 2006. Influence of chromium and cadmium on germination, seedling growth and photosynthetic pigments of soybean (Glycine max L. Merr.). Indian J. Environ. Ecoplan. 12:291-296.

Sankar Ganesh, K. 2008. Response of paddy cultivars to chromium pollution. Ph.D. Thesis, Department of Botany, Annamalai University, Annamalainagar, Tamilnadu, India.

Sankar Ganesh, K., L. Baskaran, S. Rajasekaran, K. Sumathi, Al. A. Chidambaram and P. Sundaramoorthy. 2008. Chromium stress induced alterations in biochemical and enzyme metabolism in aquatic and terrestrial plants. Coll. Surf. B Biointerfaces 63:159-163.

Sharma, G. P., R. C. Sobti, A. Chaudhary and K. K. Ahulwalia. 1988. Genotoxicity of two heavy metal compounds lead acetate and mercuric chloride in the mosquito Anapheles stephensi Liston, Cluicidae: Diptera. Cytologia 53:263-267.

Shrivastava, M. K., K. C. Jain and S. Jain. 1997. Studies on effect of phosphorus application in cotton (Gossypium hirsutum L.). Adv. Plant Sci. 12(1):279-281.

Singh, R. N. and R. F. Keefer. 1989. Uptake of nickel and cadmium by vegetables grown on soil amended with different sewage sludges. Agric. Ecol. Environ. 25:27-38.

Singh, D., K. Nath and Y. K. Sharma. 2007. Response of wheat seed germination and seedling growth under copper stress. J. Environ. Biol. 28:409414.

Williams, D. E., J. Ulamis, A. H. Pukita and J. E. Corey. 1980. Trace element accumulation, movement and distribution in the soil profile from massive applications of sewage sludge. Soil Sci. 129:119-132.

Zou, J. H., M. Wang, W. S. Jiang and D. A. Liu. 2006. Effects of Hexavalent chromium (VI) on root growth and cell division in root tip cells of Amaranthus viridis (L). Pak. J. Bot. 38(3):673-681. 